\title{
O impacto da histologia do carcinoma pulmonar na frequência das metástases ósseas*
}

\section{The Impact of Lung Carcinoma Histology on the Frequency of Bone Metastases}

\author{
Marcelo Bragança dos Reis Oliveira10 ${ }^{10}$ Larissa Costa Souza ${ }^{1}$ Ermides Javier Garcia Sampayo ${ }^{1}$ \\ Gustavo Sobral de Carvalho ${ }^{1}$ Fernanda Carvalho de Queiroz Mello ${ }^{2}$ Marcos Eduardo Machado Paschoal ${ }^{3}$ \\ ${ }^{1}$ Serviço de Traumato-Ortopedia, Universidade Federal do Rio de \\ Janeiro (UFRJ), Hospital Universitário Clementino Fraga Filho, \\ (HUCFF), Rio de Janeiro, RJ, Brasil \\ 2 Faculdade de Medicina, Universidade Federal do Rio de Janeiro \\ (UFRJ), Rio de Janeiro, RJ, Brasil \\ 3 Instituto de Doenças do Tórax, Universidade Federal do Rio de Janeiro \\ Endereço para correspondência Marcelo Bragança dos Reis Oliveira, \\ MD, PhD, Serviço de Traumato-Ortopedia, Universidade Federal do \\ Rio de Janeiro (UFRJ), Hospital Universitário Clementino Fraga Filho, \\ (HUCFF), Rio de Janeiro, RJ, 21941-590, Brasil \\ (e-mail: marceloreis@hucff.ufrj.br).
} (UFRJ), Rio de Janeiro, RJ, Brasil

Rev Bras Ortop 2019;54:524-530.

\section{Resumo \\ Palavras-chave \\ - neoplasias pulmonares \\ - metástase neoplásica \\ - histologia}

Objetivo O câncer de pulmão é a principal causa de morte por neoplasia, e os ossos são os principais locais de metástases desse tipo de câncer. O objetivo deste estudo foi avaliar a influência do tipo histológico do carcinoma de pulmão na frequência das metástases ósseas. Métodos Foram avaliados retrospectivamente os registros médicos de 407 pacientes diagnosticados com câncer de pulmão entre 2003 e 2012. A prevalência de metástases ósseas e suas associações com os subtipos histológicos foram avaliadas com o teste quiquadrado, razão de probabilidade (RP), e intervalos de confiança (IC) de 95\%. A sobrevida global foi avaliada com o método de Kaplan-Meier.

Resultados A prevalência das metástases ósseas foi de $28,2 \%(n=115)$, e a coluna vertebral foi o local mais frequente (98 metástases: $32,1 \%$ ). 0 adenocarcinoma foi o subtipo histológico mais comum de carcinoma pulmonar $(46,7 \%)$ e foi significativamente mais frequente entre os pacientes com metástases ósseas ( $13,0 \%$ versus $29,8 \%$; $p=0,0004 ; \mathrm{OR}=0,35 ; 95 \%$ IC: $0,19-0,64)$. O tempo médio de sobrevida após o diagnóstico da primeira metástase óssea foi de 4 meses.

Conclusão $\mathrm{O}$ adenocarcinoma foi o subtipo histológico mais comum de carcinoma pulmonar e foi significativamente associado a um maior risco de desenvolvimento de metástases ósseas.

\section{Abstract}

Objective Lung cancer is the leading cause of oncological deaths, and the bones are one of the most common sites of metastasis from this cancer type. The objective of this study was to evaluate the influence of lung carcinoma histology on the frequency of bone metastases.

\footnotetext{
Trabalho feito no Serviço de Traumato-Ortopedia, Universidade Federal do Rio de Janeiro, Rio de Janeiro, Brasil. Publicado Originalmente por Elsevier Ltda.
}

recebido

12 de Setembro de 2017 aceito

21 de Fevereiro de 2018
DOI https://doi.org/

10.1016/j.rbo.2018.02.002. ISSN $0102-3616$.
Copyright $(2019$ by Sociedade Brasileira License terms de Ortopedia e Traumatologia. Published by Thieme Revinter Publicações Ltda, Rio de Janeiro, Brazil 


\section{Keywords}

- lung neoplasms

- neoplasm metastasis

- histology
Methods This retrospective study evaluated the medical records of 407 patients who were diagnosed with lung carcinoma between 2003 and 2012. The prevalence of bone metastases and their association with histological subtypes were evaluated using the chi-squared test, odds ratios (ORs), and 95\% confidence intervals (Cls). Overall survival was evaluated using the Kaplan-Meier method.

Results The prevalence of bone metastases was $28.2 \%(n=115)$, and the spine was the most frequent site (98 metastases; $32.1 \%$ ). Adenocarcinoma was the most common histological subtype of lung carcinoma (46.7\%) and was significantly more frequent among patients with bone metastases ( $58.3 \%$ versus $42.1 \%$; $p=0.003$; OR $=1.92$; $95 \% \mathrm{Cl}: 1.29$ 2.97). Squamous cell carcinoma was significantly less frequent among patients with bone metastases (13.0\% versus $29.8 \% ; p=0.0004 ; \mathrm{OR}=0.35 ; 95 \% \mathrm{Cl}: 0.19-0.64$ ). The median survival time after the worst bone metastasis diagnosis was 4 months.

Conclusion Adenocarcinoma was the most common histological subtype of lung carcinoma and was significantly associated with a higher risk of developing bone metastases.

\section{Introdução}

O câncer de pulmão é uma das três malignidades mais prevalentes e é a principal causa de mortes por câncer. ${ }^{1}$ A história natural da doença inclui evolução agressiva e sobrevida reduzida. ${ }^{2,3}$ Ademais, o carcinoma metastático é o tumor ósseo maligno mais comum, ocorrendo em aproximadamente $15-40 \%$ dos pacientes com câncer de pulmão. ${ }^{4}$ Dados de necropsias mostram que a prevalência de metástases ósseas chega a $85 \%$ quando o sítio primário é o pulmão. ${ }^{5}$ Nesse contexto, os ossos são um dos locais mais frequentes de metástase de câncer de pulmão, resultando em alta morbidade e reduzida qualidade de vida entre esses pacientes. ${ }^{6-8}$

Aproximadamente $40 \%$ dos pacientes com câncer de pulmão de células não pequenas (CPCNP) desenvolvem metástases ósseas, e o adenocarcinoma é o subtipo histológico mais frequente. ${ }^{4,9-13} \mathrm{Em}$ um estudo prévio, mostramos que a histologia do câncer de pulmão influencia as características clinicopatológicas das metástases ósseas. ${ }^{14}$ Entretanto, este estudo visou a avaliar se o risco de desenvolvimento de metástases ósseas está relacionado ao subtipo histológico do carcinoma pulmonar. Esses achados podem ser úteis para guiar a vigilância precoce para a detecção de metástases ósseas ou intervenções em grupos de alto risco, a fim de melhorar a qualidade de vida e a sobrevida dos pacientes.

\section{Material e Métodos}

\section{População}

Este estudo avaliou retrospectivamente os registros médicos de 413 pacientes diagnosticados com tumores pulmonares malignos primários na nossa instituição entre 2003 e 2012. O desenho do estudo foi aprovado pelo nosso Comitê de Ética em Pesquisa. Os critérios de inclusão foram: a confirmação histopatológica de CPCNP ou de câncer de pulmão de células pequenas (CPCP) com dados de estadiamento do tumor completos. O carcinoma pulmonar foi classificado de acordo com o subtipo histológico, com os critérios da Organização
Mundial de Saúde: adenocarcinoma, carcinoma de células escamosas (CCEsc), carcinoma de células grandes (CCG), CPCNP não especificado (CPCNP/NE) e CPCP. ${ }^{15}$

Os eventos de interesse foram: ocorrência de metástases ósseas e morte. As metástases ósseas foram diagnosticadas via exame histopatológico de amostras de biópsia óssea ou via cintilografia óssea com Tc99 além de duas modalidades de exames de imagem adicionais (radiografia, tomografia computadorizada, ou imagem de ressonância magnética). As metástases ósseas foram classificadas de acordo com o tempo de diagnóstico da primeira metástase óssea em relação ao momento do diagnóstico do carcinoma pulmonar. Metástases sincrônicas foram definidas como metástases ósseas presentes no momento do diagnóstico do carcinoma pulmonar e metástases metacrônicas foram definidas como metástases ósseas que ocorreram após o diagnóstico do tumor primário. Para avaliar de forma acurada a sobrevida global, os pacientes diagnosticados com um segundo tumor maligno primário e os com data de óbito desconhecida foram excluídos. $O$ período mínimo de acompanhamento foi de 24 meses de pacientes que desenvolveram metástases ósseas (inclusive casos de óbito em menos de 24 meses).

\section{Análise Estatística}

O teste do qui-quadrado foi usado para comparar as proporções dos subtipos histológicos entre os pacientes que desenvolveram e que não desenvolveram metástases ósseas. A relação entre o subtipo histológico e a ocorrência de metástases ósseas também foram avaliadas com razão de probabilidade (RP) e intervalo de conficança de $95 \%$ (IC 95\%). A sobrevida global foi avaliada com o método Kaplan-Meier. Todas as análises foram feitas com o software SPSS for Windows, versão 10.0;(SPSS Inc., Chicago, IL, EUA) e um valor- $p<0,05$ foi considerado estatisticamente significativo.

\section{Resultados}

\section{Características dos Pacientes}

Preencheram os critérios de inclusão e foram elegíveis para análise 407 pacientes. 0 diagrama da coorte é mostrado 


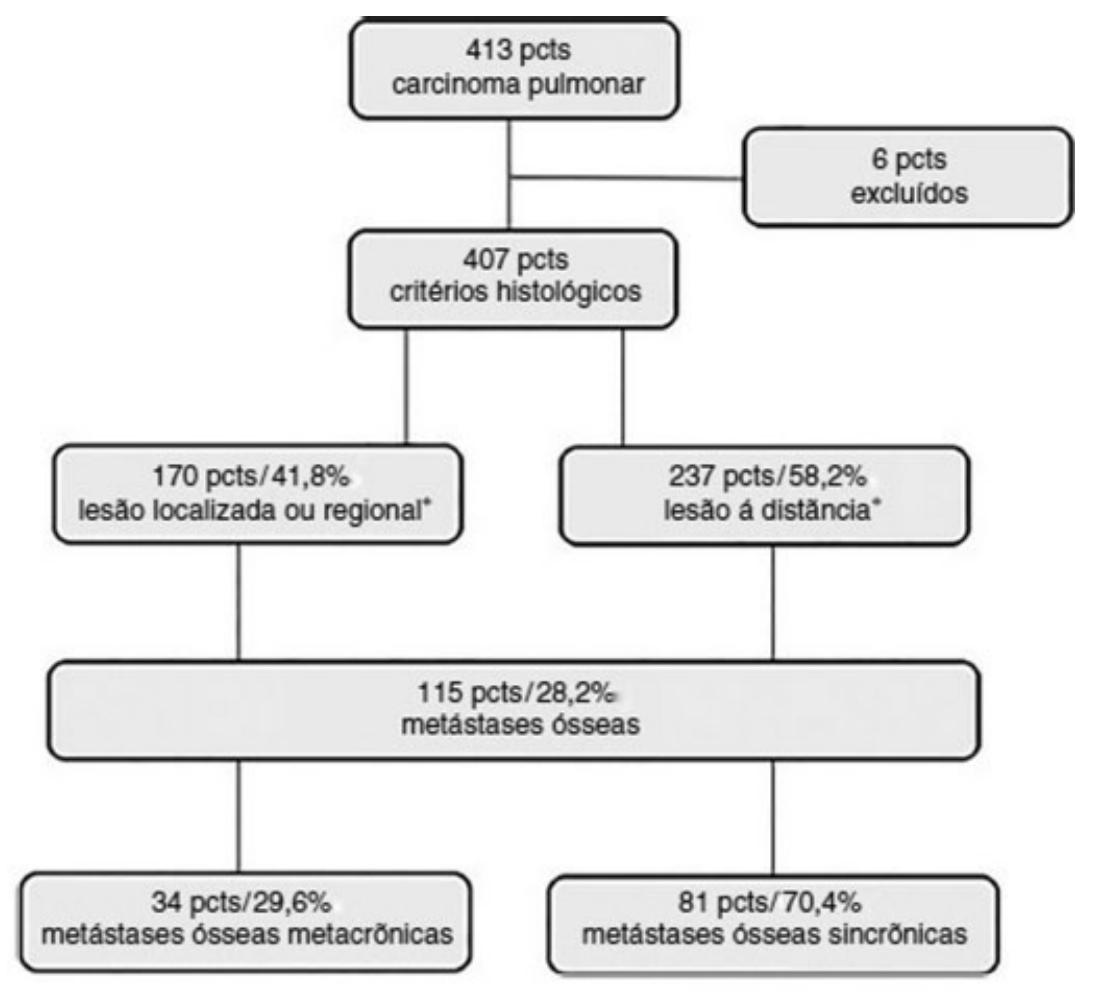

•Estágio no momento do diagnóstico

Fig. 1 Diagrama coorte. Pacientes com carcinoma pulmonar de acordo com os estágios no momento do diagnóstico e presença de metástase no momento do diagnóstico ou durante o follow-up.

na figura 1, e as características demográficas dos pacientes são mostradas na - Tabela 1. A idade média foi de 63,4 anos, e $61,4 \%$ dos pacientes eram homens. Aproximadamente $13,5 \%$ dos pacientes nunca fumaram, e os fumantes foram caracterizados como de carga tabágica $<40$ maços/ano (23,6\%) e > 40 maços/ano (62,9\%). O adenocarcinoma foi o subtipo mais comum $(n=190 ; 46,7 \%)$, seguido pelo CCEsc $(n=102 ; 25,1 \%), \mathrm{CPCNP} / \mathrm{NE}(n=56 ; 13,7 \%), \mathrm{CPCP}(n=48$; $11,8 \%)$, e CCG $(n=11 ; 2,7 \%)$ (-Tabela 1$) ; 115$ pacientes apresentaram metástases ósseas e as características dos pacientes com $(n=115)$ e sem metástases ósseas $(n=292)$ são mostradas na - Tabela 1.

\section{Prevalência de Metástases Ósseas}

A prevalência geral de metástases ósseas foi de $28,2 \%$ ( $n=$ 115). A prevalência de metástases ósseas de acordo com a histologia está representada na figura 2. As metástases ósseas foram mais prevalentes entre os pacientes com adenocarcinoma ( $n=67 ; 35,3 \%)$. O envolvimento ósseo também foi observado em pacientes com carcinoma escamocelular ( $n=15 ; 14,7 \%)$, CCG $(n=2 ; 18,1 \%)$, CNE/CPCNP $(n=16 ; 28,6 \%)$ e CPCP $(n=15 ; 31,2 \%)$. Metástases sincrônicas foram significativamente mais frequentes do que metástases metacrônicas $(n=81 ; 70,4 \%$ versus $n=34$; $29,6 \% ; p=0,0021)$.

\section{Número e Localização das Metástases}

Dentre os 115 pacientes com metástases ósseas, observamos 305 (aproximadamente 2,65 por paciente). Os sítios mais frequentes foram coluna vertebral (98 metástases; 32,1\%), cíngulo pélvico (53 metástases; 17,4\%), fêmur e úmero proximais (52 metástases; 17,1\%), e parede torácica (46 metástases; 15,1\%) (-Fig. 3).

\section{Risco de Metástases Ósseas}

A análise das proporções dos pacientes que desenvolveram ou não metástases ósseas de acordo com a histologia mostrou que o adenocarcinoma foi o subtipo histológico mais frequente em ambos os grupos, embora a frequência de adenocarcinoma tenha sido significativamente maior entre os pacientes que desenvolveram metástases ( $n=67$; $58,3 \%$ ) comparada com a dos pacientes que não desenvolveram metástases ósseas $(n=123 ; 42,1 \%)(p=0,003)$. O carcinoma celular escamoso foi significativamente o subtipo de menor frequência entre os pacientes que desenvolveram metástases ósseas ( $n=15 ; 13,0 \%$ versus $n=87 ; 29,8 \% ; p=$ $0,0004)$. Entretanto, não houve diferença estatística entre os demais subgrupos histológicos: CG $(n=2 ; 1,7 \%$ versus $n=$ $9 ; 3,1 \% ; p=0,451), \mathrm{CNE} / \mathrm{CPCNP}(n=16 ; 13,9 \%$ versus $n=40$, $13,7 \% ; p=0,954)$, е СРСР $(n=15 ; 13,0 \%$ versus $n=33$; 11,3\%; $p=0,623$ ) (-Fig. 4).

Usamos a RP e o IC de $95 \%$ para avaliar as associações entre a histologia e a ocorrência de metástases ósseas. A RP para o desenvolvimento de metástases ósseas foi de 1,92 (IC 95\% 1,29-2,97) para adenocarcinoma, 0,35 (IC 95\% 0,190,64) para CCEsc, 0,55 (IC 95\% 0,12-2,61) para CCG, 1,01 (IC 95\% 0,54-1,90) para CPCNP, e 1,17 (95\% IC 0,61-2,26) para CPCP (-Tabela 2). 
O Impacto da Histologia do Carcinoma Pulmonar Oliveira et al. 527

Tabela 1 Características gerais dos pacientes com carcinoma pulmonar e de acordo com a presença de metástases

\begin{tabular}{|c|c|c|c|c|c|c|}
\hline \multirow[t]{4}{*}{ Característica } & \multicolumn{2}{|c|}{$\begin{array}{l}\text { Pacientes com } \\
\text { carcinoma pulmonar }\end{array}$} & \multicolumn{2}{|c|}{$\begin{array}{l}\text { Pacientes com } \\
\text { carcinoma pumonar }\end{array}$} & \multicolumn{2}{|c|}{$\begin{array}{l}\text { Pacientes com } \\
\text { carcinoma pulmonar }\end{array}$} \\
\hline & \multicolumn{2}{|c|}{ Coorte geral } & \multicolumn{2}{|c|}{$\begin{array}{l}\text { Sem metastases } \\
\text { ósseas }\end{array}$} & \multicolumn{2}{|c|}{$\begin{array}{l}\text { Com metastases } \\
\text { ósseas }\end{array}$} \\
\hline & \multicolumn{2}{|c|}{$n=407$} & \multicolumn{2}{|c|}{$\mathrm{n}=292$} & \multicolumn{2}{|c|}{$n=115$} \\
\hline & $n$ & $\%$ & $\mathbf{n}$ & $\%$ & $n$ & $\%$ \\
\hline Idade média (intervalo) & \multicolumn{2}{|c|}{63,4 anos (32-87) } & \multicolumn{2}{|c|}{65,7 anos $(42-84)$} & \multicolumn{2}{|c|}{62,6 anos $(32-87)$} \\
\hline \multicolumn{7}{|l|}{ Gênero } \\
\hline Masculino & 250 & 61,4 & 175 & 59,3 & 75 & 65,2 \\
\hline Feminino & 157 & 38,6 & 117 & 40,7 & 40 & 34,8 \\
\hline \multicolumn{7}{|l|}{ Tabagismo } \\
\hline Nunca fumaram & 55 & 13,5 & 30 & 10,3 & 25 & 21,7 \\
\hline$<40$ maços/ano & 96 & 23,6 & 63 & 21,6 & 33 & 28,7 \\
\hline$\geq 40$ maços/ano & 256 & 62,9 & 199 & 68,1 & 57 & 49,6 \\
\hline \multicolumn{7}{|l|}{ Histologia } \\
\hline Adeno & 190 & 46,7 & 123 & 42,1 & 67 & 58,3 \\
\hline CCEsc & 102 & 25,1 & 87 & 29,8 & 15 & 13 \\
\hline CCG & 11 & 2,7 & 9 & 3,1 & 2 & 1,8 \\
\hline CPCNP/NE & 56 & 13,7 & 40 & 13,7 & 16 & 13,9 \\
\hline$\overline{C P C P}$ & 48 & 11,8 & 33 & 11,3 & 15 & 13 \\
\hline
\end{tabular}

Abreviações: Adeno, adenocarcinoma; CCEsc, carcinoma de células escamosas; CCG, carcinoma de células grandes; CPCNP/NE, carcinoma pulmonar de células não pequenas/não especificado; CPCP, carcinoma pulmonar de células pequenas.

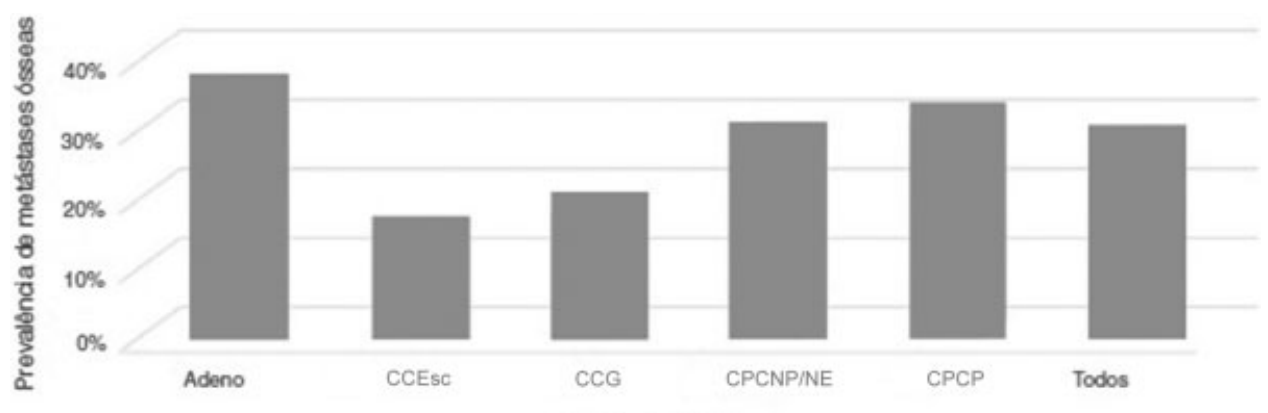

Subtipo histológico

Fig. 2 Prevalência de metástases ósseas de acordo com a histologia. Abreviações: Adeno, adenocarcinoma; CCEsc, carcinoma de células escamosas; CCG, carcinoma de células grandes; CPCNP/NE, carcinoma pulmonar de células não pequenas/não especificado; CPCP, carcinoma pulmonar de células pequenas.

\section{Sobrevida}

Os resultados para sobrevida foram calculados após a exclusão de 20 pacientes, devido ao diagnóstico de um segundo tumor primário maligno $(n=12)$ ou data desconhecida da morte $(n=$ 8). A sobrevida mediana após o diagnóstico das metástases ósseas foi de 4 meses. A sobrevida mediana para o adenocarcinoma, o CCEsc, CCG, CPCNP e CPCP foram de 3,0 meses; 4,5 meses; 9,0 meses; 2,5 meses, e 4,5 meses, respectivamente.

\section{Discussão}

Neste estudo de coorte de pacientes com carcinoma pulmonar tratados no mesmo hospital geral universitário no Brasil, observamos uma prevalência de aproximadamente $28 \%$ de envolvimento ósseo secundário. Esse resultado é semelhante aos valores previamente demonstrados e confirma que o osso é um dos principais sítios de metástase de carcinoma pulmonar. ${ }^{16}$ Estudos que compararam a frequência de sítios de metástases de CPCNP mostraram que a frequência de disseminação óssea (20-40\%) é comparável à disseminação para o fígado (25-30\%) e para o pulmão contralateral (40$50 \%$ ). ${ }^{7,10,17}$ Acreditamos que os métodos de avaliação influecniam a incidência apresentada de metástases ósseas, como estudos da década de 1990, que usaram cintilografia óssea, determinaram que a incidência era $<20 \% .{ }^{18-21}$ Por outro lado, estudos mais recentes que usaram tomografia com emissão de pósitrons demonstraram frequência de 20$40 \%{ }^{9,22,23}$ Tsuyia et al. ${ }^{10}$ identificaram $70(30,4 \%)$ pacientes 


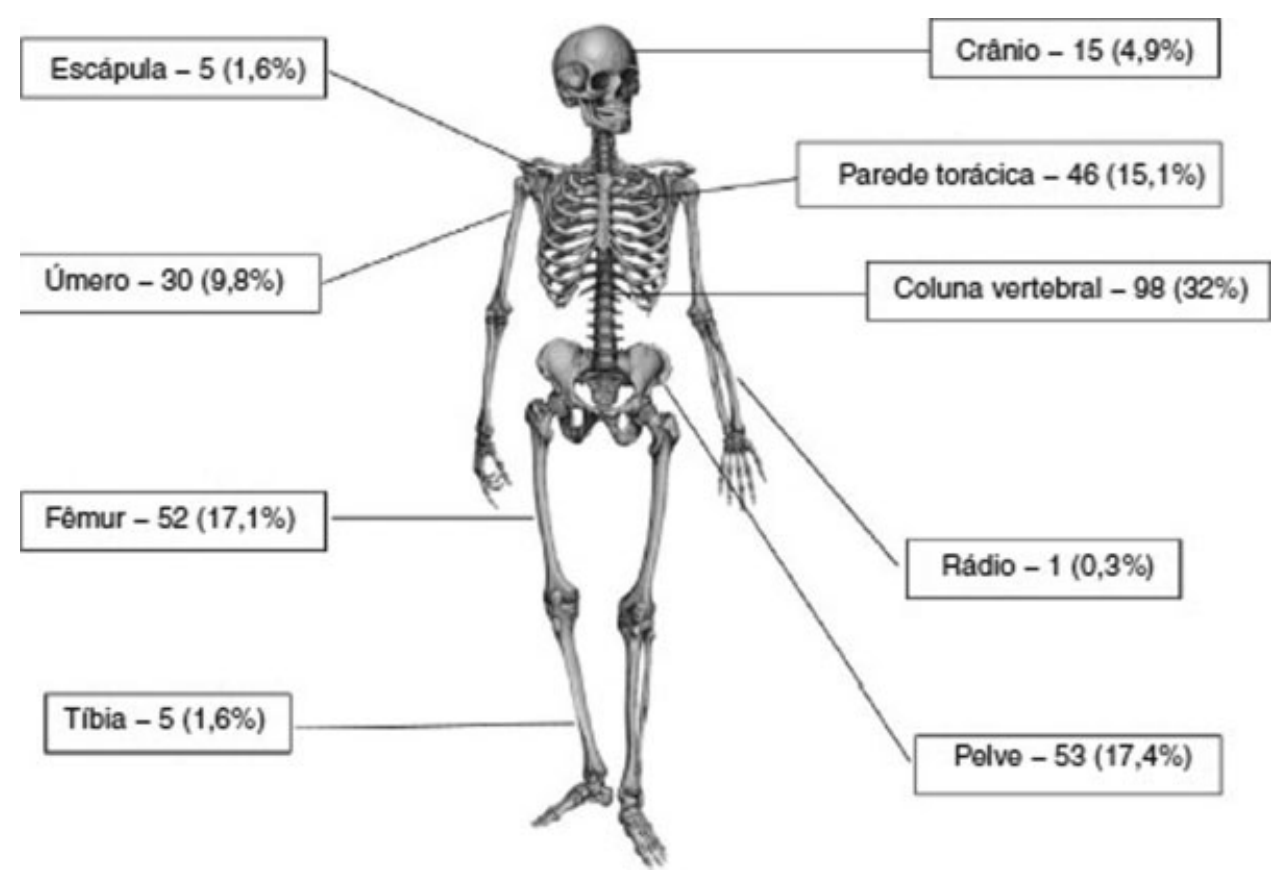

Fig. 3 Número e localização das metástases ósseas.

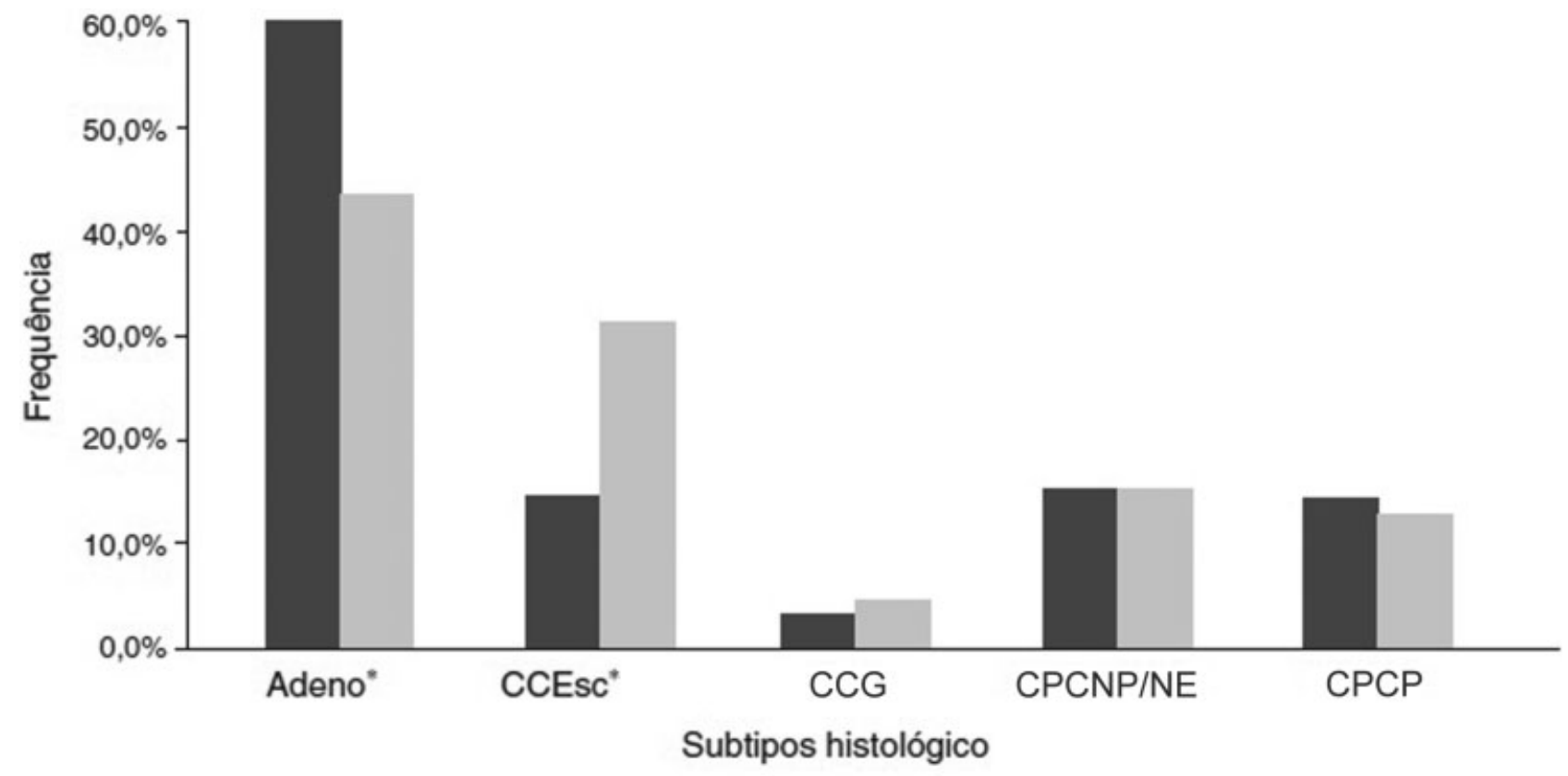

Com metástase óssea Sem metástase óssea

Fig. 4 Comparação entre a frequência dos subtipos histológicos em grupos de pacientes com carcinoma pulmonar que desenvolveram e que não desenvolveram metástases ósseas. *valor-p < 0,05. Abreviações: Adeno, adenocarcinoma; CCEsc, carcinoma de células escamosas; CCG, carcinoma de células grandes; CPCNP/NE, carcinoma pulmonar de células não pequenas/não especificado; CPCP, carcinoma pulmonar de células pequenas.

com metástases ósseas de câncer de pulmão com o uso de imagens de cintilografia, radiografia, e ressonância magnética. Seus métodos de detecção e frequência de metástases foram semelhantes aos deste estudo. Entretanto, o atual estudo incluiu uma amostragem mais ampla, dada a inclusão de paciente com CPCP.

A incidência de adenocarcinoma aumentou com o tempo e ultrapassou o CCEsc como o subtipo mais prevalente de neoplasia pulmonar. ${ }^{24}$ Neste estudo, o adenocarcinoma foi o subtipo histológico mais prevalente entre os pacientes com ou sem metástases ósseas. Nossos resultados estão de acordo com os relatórios epidemiológicos internacionais, que demonstraram o adenocarcinoma como responsável por > $45 \%$ de todos os casos. ${ }^{25}$ Entretanto, estudos na população brasileira mostraram que o CCEsc continua a ser o subtipo mais prevalente em algumas áreas do país. ${ }^{26}$ Descobrimos que 
Tabela 2 Risco de metástase óssea de acordo com a histologia do câncer de pulmão

\begin{tabular}{|l|l|l|}
\hline Histologia & $\begin{array}{l}\text { Razão de } \\
\text { probabilidade }\end{array}$ & $\begin{array}{l}\text { Intervalo de } \\
\text { confiança de 95\% }\end{array}$ \\
\hline Adeno & 1,92 & $1,29-2,97$ \\
\hline CCEsC & 0,35 & $0,19-0,64$ \\
\hline CCG & 0,55 & $0,12-2,61$ \\
\hline CPCNP/NE & 1,01 & $0,54-1,90$ \\
\hline CPCP & 1,17 & $0,61-2,26$ \\
\hline
\end{tabular}

Abreviações: Adeno, adenocarcinoma; CCEsc, carcinoma de células escamosas; CCG, carcinoma de células grandes; CPCNP/NE, carcinoma pulmonar de células não pequenas/não especificado; CPCP, carcinoma pulmonar de células pequenas.

a prevalência de metástases ósseas variou de acordo com a histologia, com maior prevalência para adenocarcinoma (35,3\%; $n=67)$ e СРCP $(31,2 \% ; n=15)$ e uma prevalência mais baixa de carcinoma celular escamoso (14,7\%; $n=15)$. Esses resultados indicam que a probabilidade de disseminação óssea pode variar de acordo com a histologia da neoplasia pulmonar. Assim, comparamos as frequências dos subtipos histológicos entre os pacientes com e sem metástases ósseas para testar essa hipótese. Nesta análise, a frequência de metástases ósseas foi significativamente e positivamente associada ao diagnóstico de adenocarcinoma (58,3\% versus $42,1 \% ; p=0,003$ ) e foi significativamente e negativamente associada ao diagnóstico de carcinoma celular escamoso (29,8\% versus $13,0 \% ; p=0,0004)$. Um estudo de coorte recente avaliou toda a população da Dinamarca e relatou maior frequência de adenocarcinoma entre pacientes com metástases ósseas $(50,3 \%)$ comparado a todos os pacientes com neoplasias pulmonares $(37,9 \%)$ e uma frequência mais baixa de carcinoma celular escamoso entre os pacientes com metástases ósseas $(13,0 \%)$ comparada a todos os pacientes com neoplasia pulmonar (24,6\%). ${ }^{11}$ Entretanto, tal estudo não avaliou a possível associação entre a histologia e a frequência das metástases. Lorusso et al. ${ }^{12}$ também relataram que o adenocarcinoma foi o subtipo histológico mais frequente, afetou $78 \%$ dos pacientes da sua amostra. De forma similar, Kagohashi et al. ${ }^{13}$ relataram que $67 \%$ de 24 pacientes com metástases ósseas tinham o subtipo adenocarcinoma. Além disso, na amostra inicial do estudo de Tsuya et al., ${ }^{10}$ o adenocarcinoma foi observado em $61 \%$ dos 259 pacientes com CPCNP, embora os autores não tenham avaliado a frequência entre os 70 pacientes que desenvolveram metástases ósseas. Previamente à nossa publicação de um estudo anterior que sugeriu que o subtipo histológico de carcinoma pulmonar poderia influenciar as características clinicopatológicas das metástases ósseas, nós havíamos definido apenas um artigo que sugeria um tropismo particular para a disseminação óssea do adenocarcinoma e que aquele estudo relatou que $45 \%$ dos adenocarcinomas desenvolviam metástases ósseas. ${ }^{21} \mathrm{~A}$ prevalência de metástases ósseas no atual estudo foi um pouco menor (35\%), embora nós tenhamos observado riscos significativamente maiores e menores de metástases ósseas de adenocarcinomas e CCEsc, respectivamente.
Muitos estudos avaliaram o envolvimento ósseo em neoplasias pulmonares sem incluir os pacientes com $\mathrm{CPCP}$, que foi o segundo subtipo mais frequente no atual estudo. Assim, permanece obscura se a frequência e outras características das metástases ósseas para esse subtipo histológico diferem daquelas de outros subtipos. Entretanto, a relativa alta frequência de metástases ósseas que observamos é compatível com a natureza mais agressiva desse câncer. ${ }^{27}$ Os estudos baseados em populações de Cetin et al. ${ }^{11}$ e Sathiakumar et al. ${ }^{28}$ relataram que as taxas de disseminação óssea foram maiores para o CPCNP. Ainda assim, os estudos nessas publicações foram alguns dos poucos que avaliaram os CPCP, diagnosticados em 16,7\% e 13\% dos pacientes com metástases ósseas, respectivamente, secundário ao carcinoma pulmonar. Essas incidências são similares aos $13 \%$ que observamos neste estudo. Por outro lado, a prevalência de metástases ósseas em $31,2 \%$ dos pacientes com CPCP foi maior em nosso estudo, comparada à prevalência de 5,8\% observada por Cetin et al. ${ }^{11} \mathrm{e}$ a prevalência de $23,3 \%$ observada por Sathiakumar et al. ${ }^{28}$ Esses resultados indicam que estudos adicionais que avaliem cuidadosamente as características das metástases ósseas desse grupo de pacientes são necessários.

Neste estudo, a maioria dos pacientes com metástases ósseas exibiu envolvimento ósseo no momento do diagnóstico da neoplasia pulmonar (70,4\%). Esses resultados podem indicar que a neoplasia pulmonar pode se espalhar nos estágios iniciais da doença. ${ }^{29}$ Entretanto, nossa frequência também pode ter sido superestimada, consequente a um possível viés de seleção, já que nossa instituição se tornou referência para pacientes com tumores ósseos em 2010, o que resultou em um aumento significativo no número de pacientes com metástases ósseas de sítios primários não determinados pelo hospital de origem.

Identificamos no presente estudo sobrevida reduzida após o diagnóstico de metástases ósseas e alguns outros estudos determinaram que a presença de metástases ósseas é um indicador de mau prognóstico para o câncer de pulmão. ${ }^{25,27,30}$ Hansen et al. $^{30}$ publicaram um relatório que considerou o efeito das metástases ósseas na sobrevida em diversos tipos de carcinomas e seus achados mostraram que pacientes com câncer de pulmão tinham uma sobrevida média de três meses após o diagnóstico das metástases ósseas. 0 tempo médio de sobrevida no atual estudo (quatro meses) foi menor do que o observado na maioria dos estudos internacionais prévios e pode estar relacionado à relativamente alta frequência de pacientes que foram diagnosticados inicialmente no estágio IV.

Nossos resultados estão mais perto dos resultados de um estudo retrospectivo francês, que estimou uma sobre- vida média de 5,8 meses. Entretanto, o desenho do nosso estudo não permite comparação de sobrevivência após o diagnóstico entre pacientes com ou sem metástases ósseas, porque o nosso objetivo foi avaliar a sobrevivência entre os pacientes diagnosticados com metástases ósseas. Além disso, o sítio pulmonar primário foi relatado como o principal fator prognóstico nos pacientes com metástases ósseas carcinomatosas. ${ }^{25,27}$ Assim, a extrema baixa sobrevida nos estudos atuais confirma três premissas: 1) Sobrevida reduzida é observada entre pacientes com neoplasia pulmonar; 2) Metástases ósseas são um preditor de mau prognóstico para qualquer carcinoma e 3) 
O fator prognóstico principal é a localização do tumor pulmonar em pacientes com carcinomas metastáticos. Entretanto, não observamos diferença estatisticamente significativa na sobrevida de acordo com a histologia.

As vantagens deste estudo são a amostra populacional brasileira maior e a inclusão do CPCP na análise da associação entre metástases ósseas e a histologia do carcinoma pulmonar. Entretanto, nossos achados apontam para a necessidade de estudos adicionais que comparem as características clinicopatológicas de acordo com a histologia. As limitações principais deste estudo incluem um possível viés de seleção, já que a institução se tornou hospital de referência para tumores ósseos em 2010 (o que aumentou a frequência de pacientes com metástases sincrônicas).

\section{Conclusão}

O adenocarcinoma foi o subtipo mais comum no grupo de pacientes com carcinoma pulmonar com ou sem metástases ósseas. Além disso, o adenocarcinoma foi associado o maior risco de desenvolvimento de metástases ósseas, enquanto que o carcinoma escamoso foi associado o menor risco. Esses dados sugerem que pacientes com adenocarcinoma podem se beneficiar de um programa de vigilância mais cauteloso com o objetivo de detectar precocemente e tratar as metástases ósseas.

\section{Conflitos de Interesse}

Os autores declaram não haver conflitos de interesse.

\section{Referências}

1 Siegel RL, Miller KD, Jemal A. Cancer statistics, 2015. CA Cancer J Clin 2015;65(01):5-29

2 Malta DC, Moura L, Souza MF, Curado MP, Alencar AP, Alencar GP. Tendência de mortalidade do câncer de pulmão, traquéia e brônquios no Brasil, 1980-2003. J Bras Pneumol 2007;33(05):536-543

3 Novaes FT, Cataneo DC, Ruiz Junior RL, Defaveri J, Michelin OC, Cataneo AJM. Lung cancer: histology, staging, treatment and survival. J Bras Pneumol 2008;34(08):595-600

4 Sugiura H, Yamada K, Sugiura T, Hida T, Mitsudomi T. Predictors of survival in patients with bone metastasis of lung cancer. Clin Orthop Relat Res 2008;466(03):729-736

5 Katagiri H, Takahashi M, Wakai K, Sugiura H, Kataoka T, Nakanishi K. Prognostic factors and a scoring system for patients with skeletal metastasis. J Bone Joint Surg Br 2005;87(05):698-703

6 Langer C, Hirsh V. Skeletal morbidity in lung cancer patients with bone metastases: demonstrating the need for early diagnosis and treatment with bisphosphonates. Lung Cancer 2010;67(01):4-11

7 Kuchuk M, Addison CL, Clemons M, Kuchuk I, Wheatley-Price P. Incidence and consequences of bone metastases in lung cancer patients. J Bone Oncol 2013;2(01):22-29

8 Avelino CU, Cardoso RM, Aguiar SS, Silva MJ. Assessment of quality of life in patients with advanced non-small cell lung carcinoma treated with a combination of carboplatin and paclitaxel. J Bras Pneumol 2015;41(02):133-142

9 Sun JM, Ahn JS, Lee S, et al. Predictors of skeletal-related events in non-small cell lung cancer patients with bone metastases. Lung Cancer 2011;71(01):89-93

10 Tsuya A, Kurata T, Tamura K, Fukuoka M. Skeletal metastases in non-small cell lung cancer: a retrospective study. Lung Cancer 2007;57(02):229-232

11 Cetin K, Christiansen CF, Jacobsen JB, Nørgaard M, Sørensen HT. Bone metastasis, skeletal-related events, and mortality in lung cancer patients: a Danish population-based cohort study. Lung Cancer 2014;86(02):247-254

12 Lorusso V, Duran I, Garzon-Rodriguez C, et al. Health resource utilisation associated with skeletal-related events in European patients with lung cancer: A subgroup analysis from a prospective multinational study. Mol Clin Oncol 2014;2(05): 701-708

13 Kagohashi K, Satoh H, Ishikawa H, Ohtsuka M, Sekizawa K. Bone metastasis as the first manifestation of lung cancer. Int J Clin Pract 2003;57(03):184-186

14 Oliveira MB, Mello FC, Paschoal ME. The relationship between lung cancer histology and the clinicopathological characteristics of bone metastases. Lung Cancer 2016;96(01):19-24

15 Travis WD, Brambilla E, Noguchi M, et al. International association for the study of lung cancer/american thoracic society/ european respiratory society international multidisciplinary classification of lung adenocarcinoma. J Thorac Oncol 2011;6 (02):244-285

16 Lee DS, Kang JH, Lee CG, et al. Predicting survival in patients with advanced non-squamous non-small cell lung cancer: validating the extent of metastasis. Cancer Res Treat 2013;45(02):95-102

$17 \mathrm{Yu} \mathrm{JL}$, Simmons C, Victor JC, et al. Impact of new chemotherapeutic and targeted agents on survival in stage IV non-small cell lung cancer. Oncologist 2011;16(09):1307-1315

18 Quint LE, Tummala S, Brisson LJ, et al. Distribution of distant metastases from newly diagnosed non-small cell lung cancer. Ann Thorac Surg 1996;62(01):246-250

19 Salvatierra A, Baamonde C, Llamas JM, Cruz F, Lopez-Pujol J. Extrathoracic staging of bronchogenic carcinoma. Chest 1990; 97(05):1052-1058

20 Michel F, Solèr M, Imhof E, Perruchoud AP. Initial staging of nonsmall cell lung cancer: value of routine radioisotope bone scanning. Thorax 1991;46(07):469-473

21 Tornyos K, Garcia O, Karr B, LeBeaud R. A correlation study of bone scanning with clinical and laboratory findings in the staging of nonsmall-cell lung cancer. Clin Nucl Med 1991;16 (02):107-109

22 Kosteva J, Langer C. Incidence and distribution of skeletal metastases in NSCLC in the era of PET. Lung Cancer 2004;46(Suppl 1): S45

23 Sekine I, Nokihara H, Yamamoto N, Kunitoh H, Ohe Y, Tamura T. Risk factors for skeletal-related events in patients with non-small cell lung cancer treated by chemotherapy. Lung Cancer 2009;65 (02):219-222

24 Janssen-Heijnen ML, Coebergh JW. The changing epidemiology of lung cancer in Europe. Lung Cancer 2003;41(03):245-258

25 Charloux A, Quoix E, Wolkove N, Small D, Pauli G, Kreisman H. The increasing incidence of lung adenocarcinoma: reality or artefact? A review of the epidemiology of lung adenocarcinoma. Int J Epidemiol 1997;26(01):14-23

26 Westphal FL, Lima LC, Andrade EC, Lima Netto JC, Silva AS, Carvalho BC. Características de pacientes com câncer de pulmão na cidade de Manaus. J Bras Pneumol 2009;35(02):157-163

27 Heidemann F, Schildt A, Schmid K, et al. Selectins mediate small cell lung cancer systemic metastasis. PLoS One 2014;9(04):e92327

28 Sathiakumar N, Delzell E, Morrisey MA, et al. Mortality following bone metastasis and skeletal-related events among patients 65 years and above with lung cancer: A population-based analysis of U.S. Medicare beneficiaries, 1999-2006. Lung India 2013;30 (01):20-26

29 Bae HM, Lee SH, Kim TM, et al. Prognostic factors for non-small cell lung cancer with bone metastasis at the time of diagnosis. Lung Cancer 2012;77(03):572-577

30 Hansen BH, Keller J, Laitinen M, et al. The Scandinavian Sarcoma Group Skeletal Metastasis Register. Survival after surgery for bone metastases in the pelvis and extremities. Acta Orthop Scand Suppl 2004;75(311):11-15 